\title{
12th International Symposium on High Pressure, Low Temperature Plasma Chemistry (HAKONE XII)
}

The 12th International Symposium on High Pressure, Low Temperature Plasma Chemistry (HAKONE XII) was organized in Trenčianske Teplice, Slovakia, from September 12 to 17, 2010 by the Department of Experimental Physics of Comenius University in Bratislava. One hundred twenty-one regular participants from 20 different countries participated in the conference and submitted 126 regular papers in 9 different topics. The next meeting, HAKONE XIII, will be organized in Poland in 2012.

In the scientific program of the conference, besides the traditionally strong topics dealing with fundamental problems and diagnostics of high pressure discharges, the fields of environmental applications and surface processes and technologies were strongly represented. Five invited lectures covered the following topics:

- patterned dielectric barrier discarges (DBDs) by J.-P. Boeuf, France;

- kinetics of electron-atom and electron-molecule processes relevant to plasmas by M. Allan, Switzerland;

- interplay of volume and surface processes in DBDs by Y. Akishev, Russia;

- nonthermal plasma activated catalysis by H.H. Kim, Japan;

- plasma sources in medicine by K.-D. Weltmann, Germany.

The International Scientific and Organisation Committee (ISOC) continued the collaboration with the European Physical Journal - Applied Physics and prepared a special issue of the journal devoted to the conference. The ISOC members preselected papers for publication in the journal and 13 of them which successfully passed the peer review procedure are presented in this issue.

All contributed papers of the HAKONE XII conference are available in the two-volume conference proceedings and on a CD distributed to all participants. More details about the HAKONE XII Symposium, the main topics and the complete scientific program can be found in: http://www.fmph.uniba.sk/hakoneXII. We would like to use this opportunity and invite the readers of the journal to the next HAKONE Symposium.

S. Matejčík Local Organizing Committee

U. Kogelschatz International Scientific and Organizing Committee HAKONE XII Chairmen 